\title{
Investigation of regularity conditions in optimal control problems with geometric mixed constraints
}

\author{
Aram V. Arutyunov, \\ Peoples' Friendship University of Russia \\ Moscow, Russia \\ E-mail: arutun@orc.ru \\ Dmitry Yu. Karamzin, \\ Universidade Estadual Paulista (IBILCE/UNESP) \\ Sao Jose do Rio Preto, SP, Brazil \\ E-mail: dmitry_karamzin@mail.ru \\ Fernando L. Pereira, \\ University of Porto, FEUP \\ Porto, Portugal \\ E-mail: flp@fe.up.pt \\ Geraldo N. Silva, \\ Universidade Estadual Paulista (IBILCE/UNESP) \\ Sao Jose do Rio Preto, SP, Brazil \\ E-mail: gnsilva@ibilce.unesp.br
}

\begin{abstract}
In this work, we study optimal control problems with mixed constraint of a general type $R(x, u, t) \in C$, where $C$ is a closed set. We undertake an effort to extend the results from [1], where, for $C$ convex, a maximum principle was derived under a weakened regularity condition. However, such a task, in its full generality, becomes rather complex, and certain obstacles appear in the proof that is not easy to overcome. Mostly, this is due to the fact that the Lagrange multiplier associated with the mixed constraints begins to take values in the convexified normal cone, but not in the normal cone itself. Another important feature is the method we use. Upon the weakening of regularity, it restricts us to consider not all closed sets but some subclass, which, for example, includes closed semi-algebraic sets.
\end{abstract}

Key words: Optimal Control, Mixed Constraints, Normal Limiting Cone

\section{Problem statement and basic definitions}

Consider the optimal control problem

$$
\left\{\begin{array}{l}
\varphi(p)+\int_{t_{1}}^{t_{2}} f_{0}(x, u, t) d t \rightarrow \min \\
\dot{x}=f(x, u, t), t \in T \\
R(x, u, t) \in C \\
p \in K
\end{array}\right.
$$

Here $T=\left[t_{1}, t_{2}\right]$ - time interval (which we assume fixed, and $t_{2}>t_{1}$ ), $\dot{x}=\frac{d x}{d t}, x$ - state variable, which takes values in the Euclidean space $\mathbb{R}^{n}, p=\left(x_{1}, x_{2}\right)$ - the so called endpoint vector, where $x_{1}=x\left(t_{1}\right), x_{2}=x\left(t_{2}\right)$, and $u \in \mathbb{R}^{m}$ is a control variable. The given vectorfunction $R: \mathbb{R}^{n} \times \mathbb{R}^{m} \times \mathbb{R}^{1} \rightarrow \mathbb{R}^{r}$ and the closed set $C$ define the geometric mixed constraints. 
The control function $u(\cdot)$ is considered measurable and essentially bounded, such that together with the $\operatorname{arc} x(\cdot)$ satisfy these mixed constraints. The set $K$ is closed and defines the endpoint constraints which have to be also satisfied for any admissible control process $(x, u)$. For the classic definition of the control problem, see in [2].

The mappings in (1),

$$
\begin{aligned}
& \varphi: \mathbb{R}^{2 n} \rightarrow \mathbb{R}^{1} \\
& f: \mathbb{R}^{n} \times \mathbb{R}^{m} \times \mathbb{R}^{1} \rightarrow \mathbb{R}^{n}, \\
& f_{0}: \mathbb{R}^{n} \times \mathbb{R}^{m} \times \mathbb{R}^{1} \rightarrow \mathbb{R}^{1}, \\
& R: \mathbb{R}^{n} \times \mathbb{R}^{m} \times \mathbb{R}^{1} \rightarrow \mathbb{R}^{r}
\end{aligned}
$$

satisfy the following main hypothesis. The maps $f, f_{0}, R$ are continuously differentiable in $(x, u)$ for a.a. $t$. On any bounded set, they and their partial derivatives in $(x, u)$ are Lebesgue measurable in $t$ for all $(x, u)$, continuous in $(x, u)$ uniformly in $t$ and bounded. The scalar function $\varphi$ is continuously differentiable.

Below we assume that the problem (1) has a solution $\left(x^{*}, u^{*}\right)$.

Consider the set-valued map

$$
U(x, t):=\left\{u \in \mathbb{R}^{m}: R(x, u, t) \in C\right\} .
$$

Definition 1 A point $u \in U(x, t)$ is said to be regular provided

$$
N_{C}(R(x, u, t)) \cap \operatorname{ker} \frac{\partial R^{*}}{\partial u}(x, u, t)=\{0\} .
$$

Here: the set $N_{C}(y)$ designates the limiting normal cone in the sense of Mordukhovich, [3]; $A^{*}$ designates the conjugate matrix or operator. The regularity means that at the considered point, the Robinson Constraint Qualification (RCQ) holds for the constraint system $R(x, u, t) \in C,[3]$. A simple example of the problem in which any feasible point $u \in U(x, t)$ is regular is given by the geometric constraint $u \in C$, or, more generally, when the matrix $\frac{\partial R}{\partial u}(x, u, t)$ has full rank.

The above condition can be reformulated in the following way: there should exist a number $\varepsilon>0$ such that

$$
\left|y \frac{\partial R}{\partial u}(x, u, t)\right| \geq \varepsilon|y| \forall y \in N_{C}(R(x, u, t)) .
$$

The upper bound of such $\varepsilon$ is also known as modulus of surjection of the constraint system $M: R(x, u, t) \in C$. The modulus of an arbitrary given constraint system $V: F(z) \in S$ we denote as $\operatorname{sur} V(z)$. Thus, the regularity of the point $u \in U(x, t)$ is equivalent to the relation

$$
\operatorname{sur} M(x, u, t)>0 .^{1}
$$

The subset of all regular points of $U(x, t)$ we denote by $U_{\text {reg }}(x, t)$. The subset of points for which sur $M(x, u, t) \geq \varepsilon$ is denoted by $U_{\mathrm{reg}}^{\varepsilon}(x, t)$. It is clear that:

$$
\begin{gathered}
U_{\mathrm{reg}}^{\varepsilon}(x, t) \subseteq U_{\mathrm{reg}}(x, t) \subseteq U(x, t) \forall \varepsilon>0, \\
U_{\mathrm{reg}}^{\alpha}(x, t) \subseteq U_{\mathrm{reg}}^{\beta}(x, t) \text { for } \alpha>\beta>0,
\end{gathered}
$$

and $U_{\text {reg }}^{0}(x, t)=U(x, t)$.

Definition 2 The trajectory $x^{*}(t)$ is said to be regular w.r.t. the mixed constraints provided there is a number $\varepsilon_{0}>0$ such that

$$
U\left(x^{*}(t), t\right) \subseteq U_{\mathrm{reg}}^{\varepsilon_{0}}\left(x^{*}(t), t\right) \text { for a.a.t } \in T .
$$

\footnotetext{
${ }^{1}$ In literature, the modulus of surjection is introduced for set-valued maps $G: X \rightarrow 2^{Y}$. If spaces $X, Y$ are finite dimensional, it is

$$
\operatorname{sur} G(x \mid y)=\inf \left\{\left|x^{*}\right|: x^{*} \in D^{*} G(x, y)\left(y^{*}\right),\left|y^{*}\right|=1\right\} .
$$

Here, $D^{*} G(x, y)$ is the limiting coderivative of $G$ at $(x, y)$, [3]. By definition, $\operatorname{sur} G(x \mid y)=\infty$ when $y \notin G(x)$. If we set $G(\cdot):=R(x, \cdot, t)-C$, then $\operatorname{sur} M(x, u, t)=\operatorname{sur} G(x, u, t \mid 0)$.
} 
For an important application case where the map $R$ is continuous in $t$ and the sets $U(t)$ are uniformly bounded, the regularity of the arc turns into the following condition:

$$
N_{C}\left(R\left(x^{*}(t), u, t\right)\right) \cap \operatorname{ker} \frac{\partial R^{*}}{\partial u}\left(x^{*}(t), u, t\right)=\{0\} \forall u \in U\left(x^{*}(t), t\right), \forall t .
$$

This condition is easy to check for a large class of sets $C$, for example for semi-algebraic sets.

Note that both set-valued maps $N_{C}(\cdot)$ and $\operatorname{ker} \frac{\partial R}{\partial u}(\cdot)$ are upper semi-continuous and thereby the regularity condition also holds in some $\delta$-tube about $x^{*}(t)$. This means that any close (in $C$-metric) to $x^{*}(\cdot)$ arc is again regular.

\section{Main Results}

Following [2], we introduce the Hamilton-Pontryagin function

$$
H(x, u, t, \psi, \lambda)=\langle\psi, f(x, u, t)\rangle-\lambda f_{0}(x, u, t) .
$$

Theorem 1 (Non-degenerate Maximum Principle) Suppose $\left(x^{*}, u^{*}\right)$ be an optimal process in problem (1). Suppose the optimal arc $x^{*}(t)$ be regular w.r.t. the mixed constraints and the sets $U(t)$ be uniformly bounded.

Then there exist a number $\lambda \geq 0$, an absolutely continuous function $\psi: T \rightarrow \mathbb{R}^{n}$, a measurable essentially bounded function $\eta: T \rightarrow \mathbb{R}^{r}$, and a constant $\kappa>0$, such that

$$
\begin{gathered}
\eta(t) \in \operatorname{conv} N_{C}(R(t)) \text { for a.a.t, } \\
\dot{\psi}(t)=-\frac{\partial H}{\partial x}(t)+\eta(t) \frac{\partial R}{\partial x}(t) \text { for a.a.t, } \\
\left(\psi\left(t_{1}\right),-\psi\left(t_{2}\right)\right) \in \lambda \frac{\partial \varphi}{\partial p}\left(p^{*}\right)+N_{K}\left(p^{*}\right), \\
\max _{u \in U(t)} H(u, t)=H(t) \text { for a.a.t, } \\
\frac{\partial H}{\partial u}(t)-\eta(t) \frac{\partial R}{\partial u}(t)=0 \text { for a.a.t, } \\
|\eta(t)| \leq \kappa(\lambda+|\psi(t)|) \text { for a.a.t, } \\
\lambda+|\psi(t)|>0 \quad \forall t \in T .
\end{gathered}
$$

Here and in sequel, if some of the arguments of a function or of a set-valued map are omitted, then it means that the extremal values $x^{*}(t), u^{*}(t), \psi(t), \lambda$ substitute the omitted arguments.

For the proof we refer the Reader to the full version paper which is to appear soon, [4]. The proof is based on a certain variational method and on the Ekeland Variational principle, [5]

Note 1 From the proof, it follows that the constant $\kappa$ in (7) depends only on the constant that bounds the the optimal process, and on the modulus of surjection $\varepsilon_{0}$.

Now proceed to the weakening of the regularity concept introduced in Definition 2.

Definition 3 The trajectory $x^{*}(\cdot)$ is said to be weakly regular w.r.t. the mixed constraints provided there is a number $\varepsilon_{0}>0$ such that

$$
u^{*}(t) \in U_{\mathrm{reg}}^{\varepsilon_{0}}\left(x^{*}(t), t\right) \text { for a.a.t. }
$$

Let $\delta$ be a positive number and $u_{0} \in U(x, t)$. Along the constraint system $M$ defining the mixed constraints in problem (1), consider the associated constraint system:

$$
M_{\delta, u_{0}}:\left\{\begin{array}{l}
R(x, u, t) \in C \\
\left|u-u_{0}\right| \leq \delta
\end{array}\right.
$$


Definition $4 A$ point $u_{0} \in U(x, t)$ is said to be proper (or, $\alpha, \gamma$-proper) provided there exist $\alpha, \gamma>0$ such that

$$
\operatorname{sur} M_{\delta, u_{0}}(x, u, t) \geq \gamma \forall u \in U(x, t):\left|u-u_{0}\right| \leq \delta, \quad \forall \delta \in(0, \alpha) .
$$

Relatively to the proper points, we shall impose the following properness condition.

Condition P) For all $\varepsilon>0 \exists \gamma>0$ s.t. for any measurable bounded selector $u(t)$ of the map $U_{\text {reg }}^{\varepsilon}\left(x^{*}(t), t\right)$ there exists a measurable scalar function $\alpha(t)$ s.t. $u(t)$ is $\alpha(t), \gamma$-proper for a.a. $t$.

In the work, we show how Condition $\mathrm{P}$ ) is wittingly satisfied when $C$ is convex, or semialgebraic, or, even, admits Whitney stratification, see [4].

Theorem 2 Let $\varepsilon \in\left(0, \varepsilon_{0}\right)$. Suppose the process $\left(x^{*}, u^{*}\right)$ be optimal in problem (1), the arc $x^{*}(t)$ be weakly regular w.r.t. the mixed constraints and Condition P) be satisfied.

Then, there exist Lagrange multipliers $\lambda, \psi, \eta$, and a number $\kappa$, which depend on $\varepsilon$, such that all the conditions of Theorem 1 hold, except the maximum condition (5) which acquires the following form:

$$
\max _{u \in \operatorname{cl} U_{\mathrm{reg}}^{\varepsilon}(t)} H(u, t)=H(t) \text { for a.a.t } \in T \text {. }
$$

In the proof, the original problem, in which the optimal trajectory $x^{*}(t)$ is weakly regular, we approximate by problems in which regularity holds in the strong sense of Definition 2, by using a certain construction based on the $\alpha, \gamma$-properness, [4].

For problems on non-fixed time interval, i.e. when $p=\left(x_{1}, x_{2}, t_{1}, t_{2}\right)$, the following result is true (as corollary of Theorem 2).

Theorem 3 Let all the assumption of Theorem 2 be satisfied. Suppose functions $R, f, f_{0}$ be continuously differentiable. Then, for any $\varepsilon \in\left(0, \varepsilon_{0}\right)$ there exist Lagrange multipliers $\lambda, \psi, \eta$, and a number $\kappa>0$, such that the conditions (2),(3), (6)-(8), (9) are satisfied, and also the following conditions hold:

$$
\begin{gathered}
\dot{h}(t)=\frac{\partial H}{\partial t}(t)-\eta(t) \frac{\partial R}{\partial t}(t) \text { for a.a.t } \\
\left(\psi\left(t_{1}\right),-\psi\left(t_{2}\right),-h\left(t_{1}\right), h\left(t_{2}\right)\right) \in \lambda \frac{\partial \varphi}{\partial p}\left(p^{*}\right)+N_{K}\left(p^{*}\right),
\end{gathered}
$$

where $h(t)=\max _{u \in \operatorname{cl} U_{\text {reg }}^{\varepsilon}(t)} H(u, t)$.

The maximum function $h(t)$ is absolutely continuous.

\section{Conclusion remarks}

1. In view of Lemma 2 from [4], Condition P) in Theorem 2 is wittingly satisfied when $C$ is convex or semi-algebraic. Therefore, for such $C$, Theorem 2 is an obvious strengthening of Theorem 1. Indeed, from the regularity assumption imposed in Theorem 1, it follows that $U(t)=U_{\text {reg }}^{\varepsilon}(t)$ for a.a. $\forall \forall \varepsilon \in\left(0, \varepsilon_{0}\right)$. However, if there exist irregular points in $U(t)$, then Theorem 1 fails to work, but Theorem 2 is still applicable under the weakened concept of regularity (Definition 3).

2. The map $U(t)$ in Theorem 2 may not be bounded unlike in Theorem 1 . This is another gain from the weakening of regularity.

3. For convex set $C$, Theorem 1 from [1] can be easily obtained from Theorem 2 by taking limit as $\varepsilon \rightarrow 0$. Indeed, in this case, the estimate (7) follows directly from (6) and weak regularity of the optimal arc. Therefore, the constant $\kappa$ in (7) will depend only $\varepsilon_{0}$, but not on the chosen $\varepsilon$. This is enough to undertake a passage to limit, which is straightforward. Thus, Theorem 2 can be regarded as an extension of Theorem 1 from [1]. Moreover, note that the concept of weak 
regularity from Definition 3 is even weaker than the corresponding notion from Definition 2 in [1]. A simple example showing that is given by the constraint $u t \leq 0$, and the control $u^{*}(t)=-t$, for $t \in[0,1]$. This control is obviously weakly regular in the sense of Definition 3 , but not in the framework of [1]. Thereby, Theorem 2 is, in fact, a strengthening of the results from [1]. Such strengthening turned out to be possible due to a more precise than in [1] work related to estimates on the modulus of surjection.

4. All the conditions of Theorem 1, except (7), could be in principle derived from Theorem 6.27 in [3], after a proper detailing. However, the estimate (7) is crucial to prove Theorem 2.

5 . The examples collected in [1] demonstrate that the strong and weak regularity conditions imposed on the optimal arc are important. Without regularity the statements of Theorems 1-3 are not already true. It is also easy to construct an example of a constraint system $R(x, u, t) \in C$ with a regular but not proper point.

There is extensive literature on mixed constraint among earlier mentioned sources. See, for example, $[6,7,8]$ and the bibliography cited therein.

\section{ACKNOWLEDGMENTS}

The research was supported by CNPq (Brazil), projects 401689/2012-3, 309335/2012-4; by the Sao Paulo State Foundation (FAPESP/CEPID), project 2013/07375-0, and by FCT (Portugal), projects Incentivo/EEI/UI0147/2013 and PEst-OE/EEI/UI0147/2011.

\section{References}

[1] A.V. Arutyunov, D.Yu. Karamzin, F.L. Pereira, Maximum Principle in Problems with Mixed Constraints under Weak Assumptions of Regularity, J. of Optimization, Volume 59, Issue 7, October 2010, pp. 1067-1083.

[2] L.S. Pontryagin, V.G. Boltyanskii, R.V. Gamkrelidze, and E.F. Mishchenko, Mathematical Theory of Optimal Processes, Moscow, Nauka, 1983.

[3] B.S. Mordukhovich, Variational Analysis and Generalized Differentiation, Springer, 2005, Vol. 1,2.

[4] A.V. Arutyunov, D.Yu. Karamzin, F.L. Pereira, G.N. Silva. Investigation of regularity conditions in optimal control problems with geometric mixed constraints. To appear.

[5] I. Ekeland, On the Variational Principle, J. Math. Anal. Appl. 47 (1974), pp. 324-353.

[6] A.V. Arutyunov. Optimality conditions: Abnormal and Degenerate Problems. Mathematics and Its Application. Kluwer Academic Publisher, 2000.

[7] A.A. Milyutin. Maximum Principle in a General Optimal Control Problem. Moscow, Fizmatlit, 2001.

[8] M.D.R. de Pinho, R.V. Vinter. Necessary conditions for optimal control problems involving nonlinear differential algebraic equations. J. Math. Anal. Appl. 212 (1997), pp. 493-516. 